\title{
Stromal tumor of unknown malignant potential of prostate: a challenge in diagnosis and management
}

\begin{abstract}
Malignancy in the Prostate is subconsciously considered to be synonymous with an Adenocarcinoma. Mesenchymal tumors of the Prostate are so rare; they often escape clinical or pathological differential diagnosis. Stromal tumor of unknown malignant potential (STUMP) of the Prostate is one such tumor. We present details of an elderly male suffering from STUMP with superadded features of a Low grade Prostatic Stromal Sarcoma (PSS) that initially posed a diagnostic challenge. This case is noteworthy not only for its rarity but also certain unusual features such as large size, pedunculated intravesical nature, prostatic central zone based and a concomitant sarcomatous transformation.
\end{abstract}

Keywords: prostate, hypercellular stroma, ureteronephrosis, chemotherapy
Volume 9 Issue 5 - 2019

\author{
Sunil Kumar, Uma Kant Dutt, Shiv Charan \\ Navriya \\ Department of Urology, AllMS Rishikesh, India
}

Correspondence: Shiv Charan Navriya, Department of Urology,AlIMS Rishikesh, India,

Email drshivnavriya2004@gmail.com

Received: October 09, 2019 | Published: October 21, 2019
Abbrevations: PSS, prostatic stromal sarcoma; STUMP, stromal tumor of unknown malignant potential; $\mathrm{BPH}$, benign hyperplasia of prostate

\section{Introduction}

Stromal tumor of unknown malignant potential of prostate is a rare tumor. Nearly about 100 case has been reported yet. We present a case of STUMP which initially posed a challenge to diagnosis to pathologist as well as urologist.

\section{Case history}

A 65-year-old man with recurrent obstructive lower urinary tract symptoms ( 3 episodes in 3 years) was referred to our hospital. Outside, he had been clinically diagnosed to have a bladder tumor and only a diagnostic transurethral resection (TUR) was attempted due to a large tumor size which was difficult to resect. The older slides were reviewed by pathologists and reported as benign hyperplasia of Prostate (BPH) in contrast to the outside clinical diagnosis of a Prostatic Adenocarcinoma.

PSA level at presentation was $1.4 \mathrm{ng} / \mathrm{ml}$. CT Urography showed a large, irregular polypoidal heterogeneously enhancing mass lesion measuring $8.7(\mathrm{ML}) \times 8(\mathrm{AP}) \times 12.1(\mathrm{CC}) \mathrm{cm}^{3}$ in dimension, arising from bladder neck/prostatic urethral area and filling the bladder almost completely. The mass lesion showed a loss of fat plane within the bladder and possible involvement of the left vesico-ureteric junction (VUJ) with left moderate hydro-ureteronephrosis as well as a $4.1 \times 4 \mathrm{~cm}$ sized exophytic component from the posterior wall (Figure 1a). On endoscopy, there was a huge tumor arising from verumontanum of the prostatic urethra and bladder neck region and was occupying almost the entire cavity of bladder. Keeping in mind the inability to endoscopically resect the tumor completely and the likely requirement of some radical procedure, only a diagnostic TUR was done which was reported as benign urothelium lined tissue with underlying benign prostatic glands. TRUS guided biopsy was done and its histopathological examination (HPE) was opined to be BPH with focal degenerative atypia. Re-resection to procure adequate amount of tumor tissue was done. Now, the HPE showed features of STUMP with prominent stromal proliferation and smudgy nuclei indicative of degenerative atypia. Transvesical enucleation of this tumor was done (Figure 1b), and the solid, white and fleshy tumor was extensively sampled in Pathology. HPE showed features of a STUMP with focal areas of increased cellularity, frank cellular atypia, increased mitotic count and occasional atypical mitotic figures (Figure 1c) that were merging gradually into the STUMP like areas. Focal, patchy necrosis was seen. Immunohistochemistry showed diffuse positivity for Vimentin and focally for PR, CD34. The tumor cells were negative for CD117, SMA, Desmin, Myogenin and ALK1. Thus, the final pathology opinion was a Low Grade PSS possibly arising in a background of STUMP.

Chemotherapy/Radical surgery was withheld due to lack of patient consent / unclear treatment protocols for the entity and the patient was kept on regular follow up with cystoscopy every 3 months. On his second follow up visit, there was evidence of a recurrence with a $1 \mathrm{~cm}$ growth in prostatic urethra MRI showed a well-defined, homogeneous, $\mathrm{T} 1$ iso and T2, PD hyper intense lesion measuring $3.2 \times 2.8 \times 2.3 \mathrm{~cm}$ is noted in the recto-vesical area in close relation to the urinary bladder and seminal vesicles with intense diffusion restriction. The lesion shows relatively homogeneous post contrast enhancement with few necrotic areas. Left aspect of proximal prostatic urethra shows a heterogeneously enhancing irregular hetero-intense lesion measuring $15 \times 12 \mathrm{~mm}$, extending into the seminal vesicles. Peripheral zone of Prostate is visualized and appears normal in signal intensity (Figure 2). No significant iliac lymphadenopathy was noted. Re-resection was done and HPE again showed features of STUMP. Thus, there appears to be evidence of recurrence in the patient. The patient is able to attend to his routine work/activities as of now. Chemotherapy / surgery needs to be planned in view of the Sarcomatous change. 


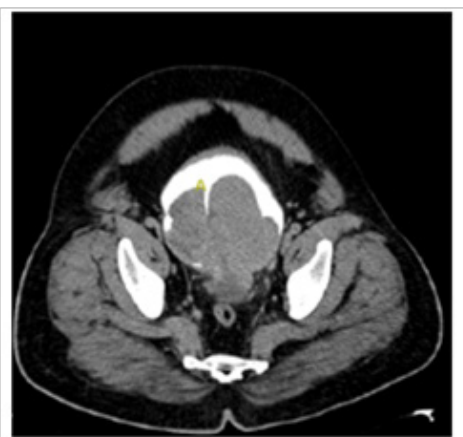

Figure 1a

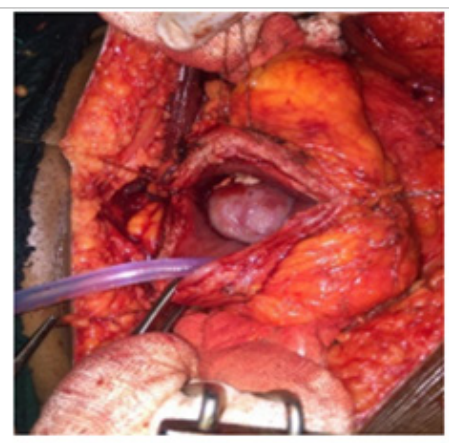

Figure 1b

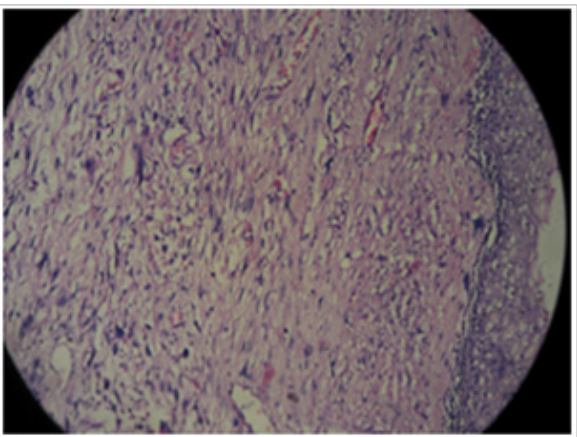

Figure 1c

Figure I (a) CECT showing a large irregular polypodial heterogeneously enhancing mass lesion involving the base of the bladder with dimensions of $8.7(\mathrm{ML})$ $x 8(\mathrm{AP}) \times 12 . \mathrm{I}(\mathrm{CC}) \mathrm{cm}^{3}$. Masslesion shows loss of fat plane with the bladder and involvement of the left VUJ. There is an exophytic component of about 4 . I $44 \mathrm{~cm}$ arising from the posterior wall as well. (b) Intraoperative images of enucleation of prostatic tumor. (c) I00X, H\&E showing part of prostatic tumor with few enlarged blotchy nuclei in an overall hypercellular stroma. Part of prostatic urethral lining epithelium seen on the right.

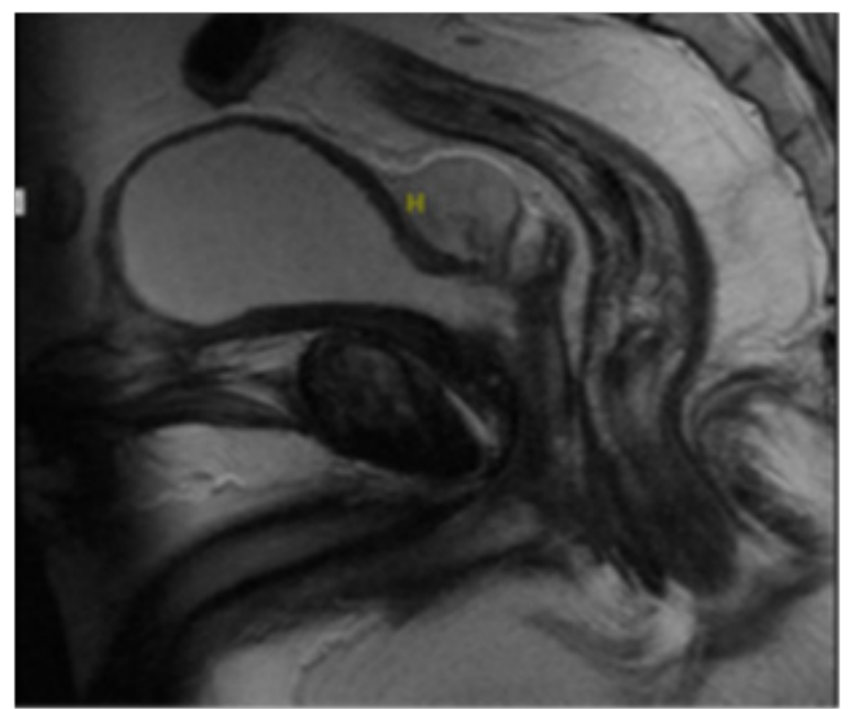

Figure 2 MRI showing a well-defined, homogeneous, TI iso and T2, PD hyper intense lesion measuring $3.2 \times 2.8 \times 2.3 \mathrm{~cm}^{3}$ in the recto-vesical area in close relation to the urinary bladder and seminal vesicles with intense diffusion restriction with relatively homogeneous post contrast enhancement and few necrotic areas.

\section{Discussion}

STUMP and even more so, PSS are rare entities with a combined total of about a 100 documented cases. ${ }^{1}$ Varied presentations include urinary obstruction, abnormal digital rectal examination, and blood in urine or semen to rectal fullness or elevated PSA levels. These tumors are variably sized and affect the transitional, peripheral zone of prostate often growing into pelvis. ${ }^{2}$ There is one report of STUMP presenting as a large intravesical mass. Gaudin et al. ${ }^{3}$ in their seminal article classified 4 morphologies of STUMP: degenerative atypia, bland hypercellular stroma, Phylloidestumor (of Breast) like stroma and myxoid predominant stroma. ${ }^{4}$ Our tumor was of the first type. For an exhaustive list of the morphologic mimics of STUMP and its immunohistochemical differentiation, the readers are referred to the article by Hansel et al. ${ }^{2}$
STUMP is notorious for recurrence in incompletely excised cases with rates reaching up to $46 \%{ }^{4}$ More worrisome is the concurrent development of a PSS in the background of STUMP (as is our case). A PSS may also arise de novo or as part of recurrence. Histopathologically, the presence of a combination of hypercellularity, cytological atypia in viable cells, atypical mitotic figures and necrosis favour sarcomatous change. Overall, PSS has a worse prognosis than STUMP due to its propensity for metastasis. ${ }^{2}$ One study demonstrated the role of chemotherapy with Ifosfamide and Doxorubicin in metastatic disease. $^{5}$

From a pathologist's perspective, an increasing reliance on core biopsies and superficial curetting available from TUR specimens, resemblance to stroma of BPH or cautery artefacts may lead to confusion and delay in diagnosis as was the case in our patient. Availability of deeper tissue in the enucleation specimen provided unequivocal evidence of classic diagnostic findings that aided the final diagnosis. Thus, adequate, deep sampling from multiple sites in large lesions is crucial.

The rarity of the entities along with their unpredictable course has hindered development of reliable treatment algorithms. There is, however a general agreement that wide excision / radical surgery such as cyst prostatectomy offer the best chance for a decent 5year survival. Depending on the general condition of the patient or the level of surgical difficulty, a wait and watch policy may also be prudent. Given, the diagnostic importance of tumoral Progesterone receptor positivity, the theranostic role of anti-progestins may be worth exploring. Our patient represents a rare entity with unusual features. A high index of suspicion with adequate biopsy may aid in an early diagnosis. The ideal treatment, as of now is adequate Surgery.

\section{Acknowledgments}

None.

\section{Funding}

None.

\section{Conflict of interest}

There is no conflict of interest. 


\section{References}

1. De Berardinis E, Busetto GM, Antonini $G$, et al. Incidental prostatic stromal tumor of uncertain malignant potential(STUMP): histopathological and immunohistochemical findings. Urologia. 2012;79(1):65-68

2. Hansel DE, Herawi M, Montgomery E, et al. Spindle cell lesions of the adult prostate. Mod Pathol. 2007;20(1):148-158

3. Wang MW, Li C, Zhang Q, et al. Prostatic stromal tumor of uncertain malignant potential presenting as a huge bladder mass: an unusual case. Asian J Androl. 2014;16(5):794-795.

4. Gaudin PB, Rosai J, Epstein JI. Sarcomas and related proliferative lesions of specialized prostatic stroma: a clinicopathologic study of 22 cases. Am J SurgPathol.1998;22(2):148-162.

5. Lam KC, Yeo W. Chemotherapy induced complete remission in malignant phyllodes tumor of the prostate metastasizing to the lung. $J$ Urol. 2002;168(3):1104-1105. 